\title{
A Case of Primary Signet-Ring Cell/Histiocytoid Carcinoma of the Eyelid:
}

Immunohistochemical Comparison with the Normal Sweat Gland and Review of the Literature Mai Iwaya, $\mathrm{MD}^{1}$, Takeshi Uehara, $\mathrm{MD}, \mathrm{PhD}^{1}$, Akihiko Yoshizawa, $\mathrm{MD}, \mathrm{PhD}^{1}$, Yukihiro Kobayashi, $\mathrm{PhD}^{1}$, Masanobu Momose, $\mathrm{PhD}^{1}$, Takayuki Honda, $\mathrm{MD}, \mathrm{PhD}^{2}$ and Hiroyoshi Ota, $\mathrm{MD}, \mathrm{PhD}^{3}$

${ }^{1}$ Department of Laboratory Medicine, Shinshu University Hospital, Matsumoto, Nagano 390-8621, Japan ${ }^{2}$ Department of Laboratory Medicine, Shinshu University School of Medicine, Matsumoto, Nagano 390-8621, Japan

${ }^{3}$ Department of Biomedical Sciences, School of Health Sciences, Shinshu University School of Medicine, Matsumoto, Nagano 390-8621, Japan

\section{Address correspondence to:}

Hiroyoshi Ota, Department of Biomedical Sciences, School of Health Sciences, Shinshu University School of Medicine,

\section{3-1-1 Asahi,}

Matsumoto 390-8621, Japan

Tel\&Fax: +81-263-37-2389

E-mail: hohta@ shinshu-u.ac.jp

This work was supported by a Grant-in-Aid for Scientific Research C-23590664 (to HO) from the Ministry of Education, Culture, Sports, Science and Technology, Japan.

Disclosure: There are no conflicts of interest to declare.

\begin{abstract}
Primary signet-ring cell/histiocytoid carcinomas of the eyelid are extremely rare tumors considered to originate from sweat glands. Here, we report the case of a 72-year-old man diagnosed with primary signet-ring cell/histiocytoid carcinoma of the eyelid and present immunohistochemical analyses of the eyelid apocrine gland
\end{abstract}


(Moll gland) and apocrine and eccrine sweat glands of perineum and axilla. Widespread infiltration of tumor cells with signet-ring cell or histiocytoid appearance was observed in his left eyelid, orbit, and periocular lesion. Tumor cells expressed mucins and showed immunoreactivity that was similar to that of the Moll gland: MUC6(+), GlcNAc $\alpha 1 \rightarrow 4$ Gal $\rightarrow \mathrm{R}(-)$, MUC2(-), MUC5AC(-), GCDFP15(+), CD15(+), S100(-), CK7(+), CK20(-), ER(+), PgR (+), HER2(-), E-cadherin(+), p63(-), PSA (-), and TTF-1(-). The tumor cells differed from those of perineal and axillary apocrine and eccrine sweat glands, which were MUC6(-). The Moll gland was ER(-) and PgR(-), whereas perineal and axillar apocrine sweat glands were $\operatorname{ER}(+)$ and $\operatorname{PgR}(+)$, and perineal and axillary eccrine sweat glands were $\mathrm{ER}(+)$ and $\operatorname{PgR}(-)$. The tumor showed characteristics similar to that of the eyelid Moll gland, which is demonstrated to be an apocrine gland with a protein expression distinct from that of other apocrine glands. MUC6 and GCDFP15 expression are useful in identifying the Moll's gland immunophenotype and GCDFP15, ER and PgR expression are useful in distinguishing primary eyelid signet ring/histocytoid carcinoma from gastrointestinal malignancies.

Keywords: eyelid, immunohistochemistry, mucin, sweat gland carcinoma 


\section{Introduction}

Primary signet-ring cell/histiocytoid carcinoma of the eyelid is a rare tumor, and to date, only 28 cases have been reported. ${ }^{1,2}$ The tumor develops mostly in elderly men, and the typical clinical manifestation is a progressive painless swelling of the affected eyelid, leading to a monocle-like appearance. The tumor cells show signet-ring cell or histiocytoid appearance and diffuse infiltration into the dermis and subcutis, without epidermal involvement. The tumor is considered to originate from sweat glands in the eyelid: ${ }^{1-5}$ eccrine and apocrine sweat glands (Moll gland); however, there is much debate on whether the tumor expresses the phenotype of eccrine or apocrine sweat glands. The tumor mimics metastasizing signet ring cell adenocarcinoma, especially from the gastrointestinal tract, breast, and urinary bladder. ${ }^{1-5}$

Here, we report a patient with primary signet-ring cell/histiocytoid carcinoma of the eyelid who showed a phenotype of protein expression similar to that of Moll gland and investigate its histogenesis by immunohistochemical comparison of the tumor with normal apocrine and eccrine sweat glands of the perineal and axillary region.

\section{Case report}

A 72-year-old Japanese man presented to our hospital with swelling of his left eyelid (Fig. 1A) that had developed over the past 6-7 months. Physical examination showed a diffuse induration of his left eyelid and focal involvement of the periorbital skin. His medical history was unremarkable, except for diabetes and hyperlipidemia. Maxillary and facial computed tomography and magnetic resonance imaging were performed, and they showed diffuse infiltration of the left orbit and periocular region (Fig. 1B). A biopsy of the periorbital skin revealed infiltration of signet-ring cells and histiocyte-like cells.

Gastrointestinal endoscopic examination showed multiple gastric ulcers. Biopsy specimens of the lesions revealed reactive epithelial changes and inflammatory cell infiltrates. The patient's serum carcinoembryonic

antigen and carbohydrate antigen $19-9$ levels were within the normal range. ${ }^{18} \mathrm{~F}$-fluorodeoxy glucose positron emission tomography showed a marked accumulation of the glucose radiotracer in his left orbit and periocular lesion, and there were no other significant sites of accumulation to indicate an occult primary tumor or 
metastatic lesions.

Left orbital exenteration was performed, and the patient underwent adjuvant radiotherapy (a total of 70 Gy) and chemotherapy with TS-1that contains tegafur, gimeracil and oteracil potassium. Within 6 months, the tumor recurred as multiple cervical lymph-node metastases. One and a half years after the left orbital exenteration, the patient is alive with the disease.

\section{Materials and Methods}

The tissue specimens were fixed in $10 \%$ buffered formalin and embedded in paraffin. Sections of $3-\mu \mathrm{m}$ thickness were cut and stained with hematoxylin-eosin and diastase digestion-Alcian blue (pH2.5)-periodic acid-Schiff (Di AB-PAS) and immunohistochemically studied by using the immunoenzyme polymer method (Histofine Simple Stain MAX PO Multi, Nichirei Biosciences, Tokyo, Japan) or by using an automated stainer for immunostaining for HER2, ER, and PgR (Ventana Medical Systems, Tucson, AZ) with 3, 3'-diaminobenzidine as the chromogen. The commercially available antibodies are listed in Table 1.

We used 4 cases of resected eyelid specimens containing Moll gland and 5 cases of resected perineal skin and 3 cases of resected axillary skin containing histologically normal apocrine sweat glands and eccrine sweat glands as control specimens. These control specimens were retrieved from the pathology files at Shinshu University Hospital.

The results of immunostaining were evaluated as the percentage of positively stained cells. The degree of positive staining was graded into 4 categories as follows: (i) -, negative, no positive cells; (ii) +, focal expression, less than one third of the cells were positive; (iii) ++ , one third to two thirds of the cells were positive; and (iv) +++ , strong diffuse expression, more than two thirds of the cells were positive.

\section{Acknowledgements}

This work was supported by a Grant-in-Aid for Scientific Research C-23590664 (to HO) from the Ministry of Education, Culture, Sports, Science and Technology, Japan. 


\section{Results}

\section{Tumor of the eyelid}

\section{Histopathologic findings}

Microscopic examination of the resected specimen revealed widespread infiltration of the tumor. The tumor extended across the upper and lower eyelid, periorbital skin, and orbit. Infiltrative growth from the upper dermis into subcutaneous tissue, skeletal muscle, and orbital soft tissue was detected, but the epidermis and conjunctival epithelium were not involved.

In the dermis and subcutis, the tumor cells were scattered among the collagen fibers as single cells (Fig. 2). The cells were atypical, with abundant clear or eosinophilic cytoplasm, and resembled histiocytes (Fig. 2). Some cells contained single vacuoles and had a characteristic signet-ring appearance (Fig. 2). In the orbital soft tissue, the tumor cells formed a solid diffuse growth and had a monomorphic appearance. The cells were larger than those in the dermis and subcutis and had relatively large nuclei and abundant amphophilic cytoplasm resembling that of histiocytes (Fig. 3).

\section{Histochemical and immunohistochemical findings}

Table 2 summarizes the immunohistochemical profiles observed in the tumor of the eyelid.

The intracytoplasmic vacuoles showed positive results for staining with Di-AB/PAS (Fig. 2B). The tumor cells showed diffusely positive results for CK7 (Fig. 2C) and CD15 and focally positive results for GCDFP-15 (Fig. 2D), MUC6, (Fig. 2E), E-cadherin, ER (Fig. 3B), and PgR. CK20, S-100, HER2, GlcNAc $\alpha 1 \rightarrow 4$ Gal $\rightarrow$ R (Fig. 2F), MUC2, MUC5AC, p63, PSA, and TTF-1 were all negative.

\section{Normal Moll glands, perineal and axillary apocrine sweat glands, and eccrine sweat glands \\ Histochemical and immunohistochemical findings}

Moll glands and perineal and axillary sweat glands (Fig. 4) revealed a positive reaction with Di-AB-PAS at the luminal surfaces of the acinal cells (Fig. 4B). Table 2 summarizes the immunohistochemical reactivity of normal Moll glands, perineal and axillary apocrine/eccrine sweat glands. 
Briefly, CK7 was expressed in Moll glands (Fig. 4C1), and perineal and axillary apocrine (Fig. 4C2)/eccrine glands (Fig. 4C3), but CK20 was not expressed in these glands. GCDFP-15 and CD15 were expressed in Moll glands (Fig. 4D1), and perineal and axillary apocrine (Fig. 4D2)/eccrine sweat glands (Fig. 4D3). Acinal cells and myoepithelial cell of perineal and axillary eccrine glands showed positive results for S100. E-cadherin was expressed in the sweat glands. ER was expressed in the perineal and axillary apocrine (Fig. 4E2) /eccrine sweat glands (Fig. 4E3) but not in the Moll glands (Fig. 4E1). PgR was expressed only in perineal and axillary apocrine sweat glands, and HER2 was expressed only in perineal and axillary eccrine sweat glands. MUC6 was expressed in the Moll gland (Fig. 5F1), but not in perineal and axillary apocrine/eccrine sweat glands. GlcNAc $\alpha 1 \rightarrow 4 \mathrm{Gal} \rightarrow \mathrm{R}$ (Fig. 5G1), MUC2, and MUC5AC were all negative. p63 was expressed in myoepithelial cells but not in acinal cells. 


\section{Discussion}

Histologically, primary signet-ring cell/histiocytoid carcinoma of the eyelid may be indistinguishable from signet-ring cell carcinoma of the gastrointestinal tract and histiocytoid lobular carcinoma of the breast; these are the most common origins for secondary metastatic tumors of the eyelid ${ }^{6}$ and may be detected prior to the primary tumor. ${ }^{1-3}$ Thus, before making a diagnosis of primary signet-ring cell/histiocytoid carcinoma of the eyelid, close clinicopathological correlation and a systemic work-up should be performed to exclude a metastatic primary malignancy origin.

The immunohistochemical analysis showed that the tumor cells exhibited positive results for CK7, GCDFP-15, CD15, ER, and PgR. Immunoreactivity for E-cadherin was weak. In addition, the tumor cells showed negative results for S-100 protein, CK20, p63, MUC2, and MUC5AC. These results are consistent with those of earlier reports. A novel finding of the present study was that, in the present case, positive results were observed for MUC6, whereas negative results were observed for HIK1083-reactive mucin, which is co-expressed with MUC6 in the gastric-gland mucous cells, Brunner gland, and accessory glands around the bile duct and pancreatic duct. This mucin expression pattern is identical to that observed in the Moll gland and to that previously reported in normal breast and breast carcinoma specimens. ${ }^{7}$ Therefore, no conclusions about the primary or metastatic nature of the eyelid neoplasms can be made from this mucin immunoprofile. Significantly, GCDFP-15 has been reported to be expressed in the apocrine and eccrine sweat glands, but not in the normal gastrointestinal tract. ${ }^{8}$ Furthermore, gastric adenocarcinomas show negative results for ER and PgR. ${ }^{8}$ This expression pattern of GCDFP-15, ER, and PgR in the present case can exclude the possibility of metastasis of gastrointestinal cancer to the eyelid.

There is much debate on the histogenesis of primary signet-ring cell/histiocytoid carcinoma of the eyelid. It has been postulated to originate from the apocrine sweat gland ${ }^{3,9}$ or the eccrine sweat gland., ${ }^{4,5,10,11}$ Primary cutaneous carcinoma with features of signet-ring cell/histiocytoid carcinoma has been reported in the axilla ${ }^{12}$ as well as in the eyelid. The most common sites of occurrence for this tumor are the apocrine sweat glands, supporting an apocrine differentiation of this tumor. In addition, our patient expressed MUC6 that was detected in the Moll gland but not in the eccrine sweat gland, suggesting that the tumor cells expressed the phenotype of 
the Moll gland.

The present case revealed typical clinical features. The clinical course of the primary signet-ring cell/histiocytoid carcinoma of the eyelid is indolent, but it extends to the adjacent eyelid, involves the adjacent orbit, and often recurs over many years with metastasis to the regional lymph node ${ }^{1,10,11}$ and internal viscera. ${ }^{1,2,5}$ Furthermore, there have been reports of patients who have died of widespread metastasis of this disease. ${ }^{13,14}$

From an evolutionary perspective, 2 different scenarios have been proposed for the evolution of the apocrine sweat glands; ${ }^{15}$ the first scenario is that apocrine sweat glands primarily function in reproduction and sexual behavior, and the second scenario is that they are primarily a defense against microorganisms. Indeed, 2 different phenotypes of apocrine sweat glands corresponding to these 2 proposed functions exist. Glands thought to have evolved to function in reproduction and sexual behavior express ER and develop during puberty and become atrophic in old age (for example, the apocrine axillary glands). Glands proposed to have a primary antimicrobial function develop in infants and do not express ER (for example, the Moll gland in the eyelid and the ceruminous glands in the ear canal). In this study, we also detected ER in the perineal and axillary apocrine gland but not in the Moll gland. In addition, we observed MUC6 expression in the Moll gland but not in the perineal and axillary apocrine gland. These differences could be related to the different functions of these 2 types of apocrine glands.

In conclusion, we have reported a case of primary signet-ring cell/histiocytoid carcinoma of the eyelid. Histologic and immunohistochemical analyses indicated that the tumor showed characteristics similar to that of the Moll gland, which is demonstrated to be an apocrine gland with a protein expression distinct from that of other apocrine glands. GCDFP15 and MUC6 expression are useful in identifying the Moll's gland immunophenotype and GCDFP15, ER and PgR expression are useful in distinguishing primary eyelid signet ring/histocytoid carcinoma from gastrointestinal malignancies. 


\section{Figure legends}

Figure 1

A 72-year-old man with a diffuse induration of his left eyelid and focal involvement of the periorbital skin that produced a monocle-like appearance (A).

Magnetic resonance image showing diffuse infiltration of the left orbit and periocular region (B).

Figure 2

Histology, histochemistry, and immunostaining of signet-ring cell/histiocytoid carcinoma of the eyelid in the dermis and subcutis.

Single tumor cells were scattered among the collagen fibers. The cells were atypical, with eosinophilic cytoplasm, and resembled histiocytes. Some cells contained single vacuoles and exhibited a signet-ring cell appearance (A). The intracytoplasmic vacuoles showed positive results for staining with diastase digestion-Alcian blue (pH 2.5) and periodic acid Schiff reaction (diAB-PAS) (B). The tumor cells showed positive results for CK7 (C), GCDFP-15 (D), and MUC6 (E), but not for GlcNAc $1 \rightarrow 4 \mathrm{Gal} \rightarrow \mathrm{R}$, as defined by the absence of immunoreactivity with antibody HIK1083 (F).

Figure 3

Histology and immunostaining of signet-ring cell/histiocytoid carcinoma of the eyelid in the orbital soft tissue. The tumor cells were larger than those in the dermis and subcutis and had relatively large nuclei and abundant amphophilic cytoplasm, causing them to resemble histiocytes (A), and showed positive results for ER (B, immunostaining for ER).

Figure 4

Histology, histochemistry, and immunostaining of Moll glands, perineal apocrine sweat glands, and perineal sweat glands.

Hematoxylin and eosin staining (HE), diastase digestion-Alcian blue (pH 2.5) and periodic acid Schiff reaction (diAB-PAS), and immunostaining of normal sweat glands. Each column of panels represents normal sweat glands, in the order from left to right, as follows: Moll gland, perineal apocrine sweat gland, and perineal eccrine sweat gland. Each row of panels represents an immunostaining result, from top to bottom, as follows: HE (A), 
diAB-PAS(B), cytokeratin 7 (CK7) (C), gross cystic disease fluid protein-15 (GCDFP15) (D), estrogen receptor $(\mathrm{ER})(\mathrm{E})$, MUC6 $(\mathrm{F})$, and GlcNAc $\alpha 1 \rightarrow 4 \mathrm{Gal} \rightarrow \mathrm{R}(\mathrm{G})$ Acinal cells of sweat glands revealed a positive reaction with AB-PAS at the luminal surface (B), CK (C), and GCDFP-15 (D). Perineal sweat glands (E2 and E3), but not (E1), showed positive results for ER. MUC6 was expressed only in the Moll glands (F1). GlcNAc $\alpha 1 \rightarrow 4 \mathrm{Gal} \rightarrow \mathrm{R}$ was not expressed in the Moll glands (G1) or the perineal glands (G2 and G3). 


\section{References}

1. Requena L, Prieto VG, Requena C, et al. Primary signet-ring cell/histiocytoid carcinoma of the eyelid: a clinicopathologic study of 5 cases and review of the literature. Am J Surg Pathol. 2011;35:378-391.

2. Mortensen AL, Heegaard S, Clemmensen O, et al. Signet ring cell carcinoma of the eyelid - the monocle tumour. APMIS. 2008;116:326-332.

3. Langel DJ, Yeatts RP, White WL. Primary signet ring cell carcinoma of the eyelid: report of a case demonstrating further analogy to lobular carcinoma of the breast with a literature review. Am $J$ Dermatopathol. 2001;23:444-449.

4. Swinson B, Ryan F, Barrett AW, et al. Histiocytoid eccrine sweat gland carcinoma of the eyelid: report of a case. Clin Exp Dermatol. 2006;31:786-789.

5. Kim YM, Kim JW, Oh DE. A case of histiocytoid variant eccrine sweat gland carcinoma of the orbit. Korean journal of ophthalmology : KJO. 2011;25:54-56.

6. Steinbrecher JS, Silverberg SG. Signet-ring cell carcinoma of the breast. The mucinous variant of infiltrating lobular carcinoma? Cancer. 1976;37:828-840.

7. Nakajima K, Ota H, Zhang MX, et al. Expression of gastric gland mucous cell-type mucin in normal and neoplastic human tissues. J Histochem Cytochem. 2003;51:1689-1698.

8. Park SY, Kim BH, Kim JH, et al. Panels of immunohistochemical markers help determine primary sites of metastatic adenocarcinoma. Arch Pathol Lab Med. 2007;131:1561-1567.

9. Jakobiec FA, Austin P, Iwamoto T, et al. Primary infiltrating signet ring carcinoma of the eyelids. Ophthalmology. 1983;90:291-299. 
10. Wollensak G, Witschel H, Bohm N. Signet ring cell carcinoma of the eccrine sweat glands in the eyelid. Ophthalmology. 1996;103:1788-1793.

11. Kramer TR, Grossniklaus HE, McLean IW, et al. Histiocytoid variant of eccrine sweat gland carcinoma of the eyelid and orbit: report of five cases. Ophthalmology. 2002;109:553-559.

12. Misago N, Shinoda Y, Okawa T, et al. Histiocytoid and signet-ring cell carcinoma of the axilla: a type of cutaneous apocrine carcinoma equivalent to histiocytoid lobular carcinoma of the breast? Clin Exp Dermatol. 2011;36:874-877.

13. Bellman B, Gregory NA, Silvers D, et al. Sweat gland carcinoma with metastases to the skin: response to 5-fluorouracil chemotherapy. Cutis. 1995;55:221-224.

14. Thomas JW, Fu YS, Levine MR. Primary mucinous sweat gland carcinoma of the eyelid simulating metastatic carcinoma. Am J Ophthalmol. 1979;87:29-33.

15. Stoeckelhuber M, Stoeckelhuber BM, Welsch U. Human glands of Moll: histochemical and ultrastructural characterization of the glands of Moll in the human eyelid. J Invest Dermatol. $2003 ; 121: 28-36$. 
Table 1. List of primary antibodies used in this study

\begin{tabular}{|c|c|c|c|c|}
\hline Antigen & Clone & Source & Dilution & Retrieval \\
\hline CK7 & OV-TL $12 / 30$ & $\begin{array}{l}\text { DAKO } \\
\text { (Carpinteria, CA) }\end{array}$ & $1: 30$ & Microwave \\
\hline CK20 & Ks20.8 & DAKO & $1: 30$ & Microwave \\
\hline GCDFP-15 & D6 & $\begin{array}{l}\text { COVANCE } \\
\text { (Princeton, NJ) }\end{array}$ & $1: 30$ & Microwave \\
\hline $\mathrm{CD} 15$ & MMA & $\begin{array}{l}\text { BD Biosciences } \\
\text { (Franklin Lakes, NJ) }\end{array}$ & $1: 30$ & Microwave \\
\hline S-100 & Rabbit polyclonal & DAKO & $1: 800$ & Microwave \\
\hline E-cadherin & 36 & BD Biosciences & $1: 2000$ & Microwave \\
\hline ER & SP1 & $\begin{array}{l}\text { Ventana Medical Systems } \\
\text { (Tucson, AZ) }\end{array}$ & $1: 1$ & Microwave \\
\hline PgR & $1 \mathrm{E} 2$ & Ventana & $1: 1$ & Microwave \\
\hline HER2/ neu & $4 \mathrm{~B} 5$ & Ventana & $1: 1$ & Microwave \\
\hline MUC2 & Ccp58 & $\begin{array}{l}\text { Novocastra } \\
\text { (Newcastle-upon-Tyne, UK) }\end{array}$ & $1: 100$ & Microwave \\
\hline MUC5AC & CLH2 & Novocastra & $1: 100$ & Microwave \\
\hline MUC6 & CLH5 & Novocastra & $1: 100$ & Microwave \\
\hline GlcNAc $\alpha 1 \rightarrow 4 \mathrm{Gal} \rightarrow \mathrm{R}$ & HIK1083 & $\begin{array}{l}\text { Kanto Reagents } \\
\text { (Tokyo, Japan) }\end{array}$ & $1: 10$ & Microwave \\
\hline $\mathrm{P} 63$ & $4 \mathrm{~A} 4$ & $\begin{array}{l}\text { Nichirei Bioscience Inc. } \\
\text { (Tokyo, Japan) }\end{array}$ & $1: 1$ & Microwave \\
\hline PSA & $35 \mathrm{H} 9$ & Novocastra & $1: 40$ & Microwave \\
\hline TTF-1 & 8G7G3/1 & DAKO & $1: 100$ & Microwave \\
\hline
\end{tabular}


Table 2. Immunohistochemical expression patterns observed in primary signet-ring cell/histiocytoid carcinoma of the eyelid and acinal cells of normal Moll glands, and perineal and axillary apocrine/eccrine sweat glands

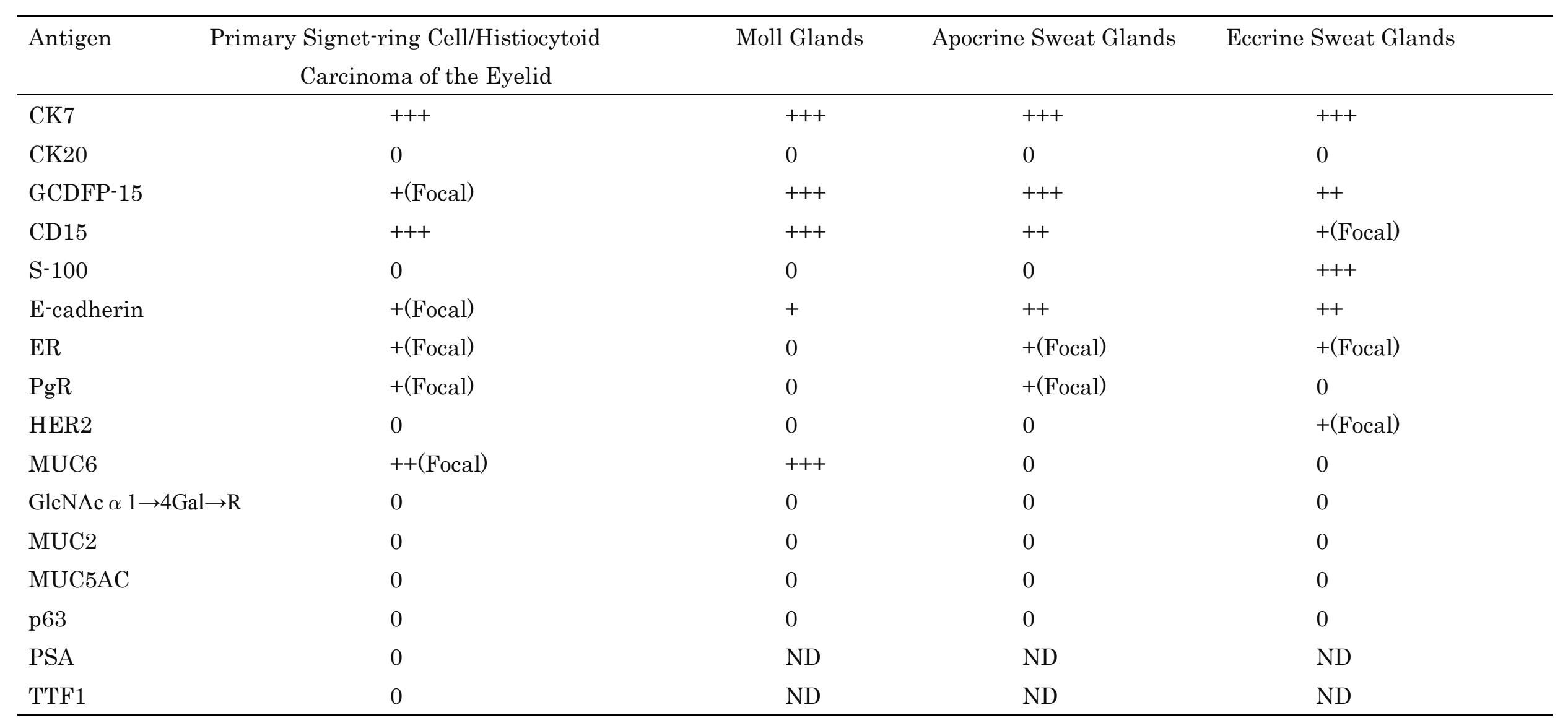

The intensity of immunostaining was scored as follows: 0 , negative; +, weak; ++, moderate; +++, strong; and ND, not done. 


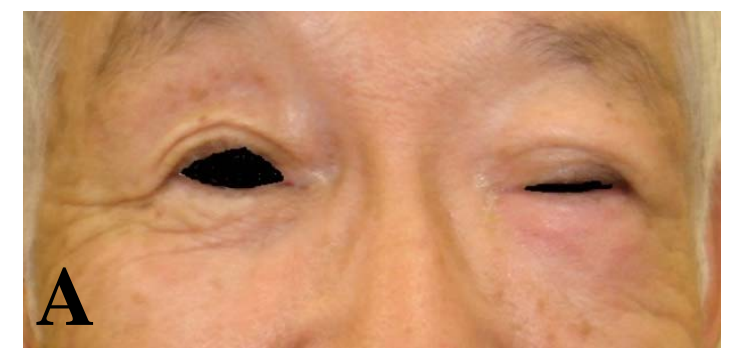

Fig 1 B 1 Antor 


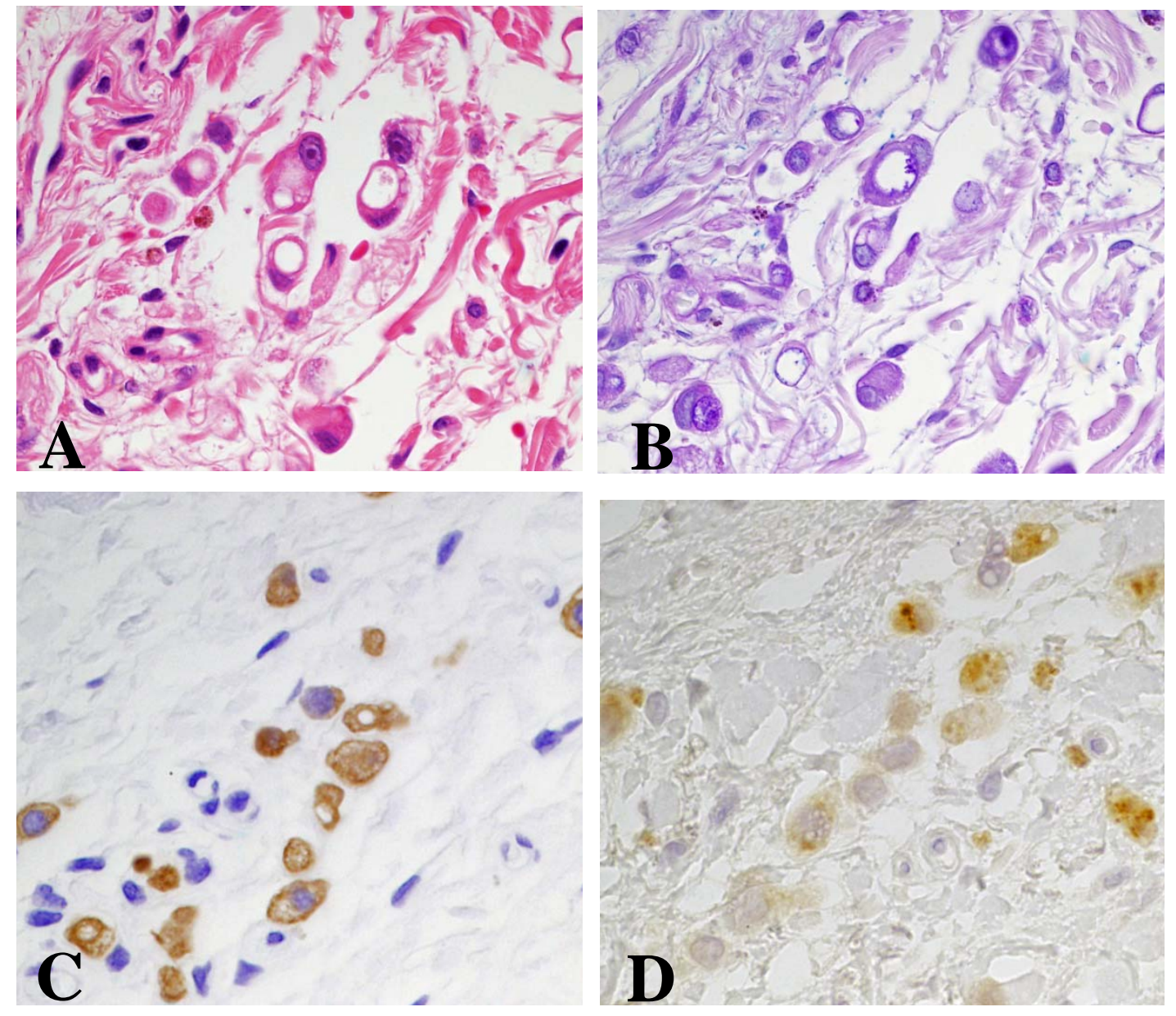

Fig 2
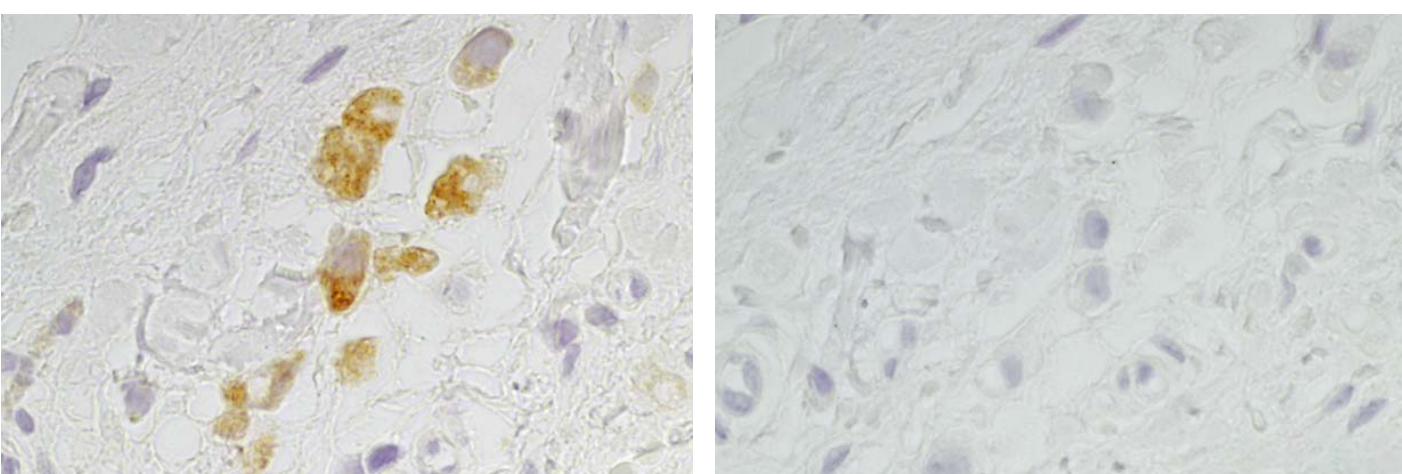

$$
\text { E : }
$$

\section{F}
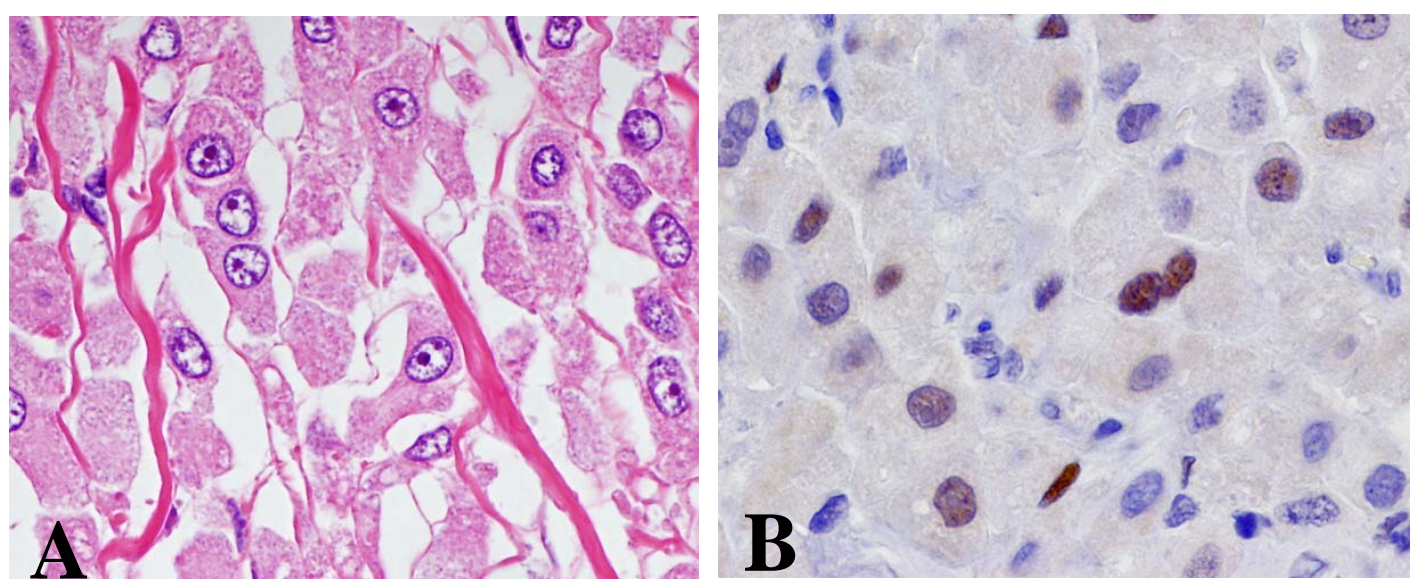
Moll gland
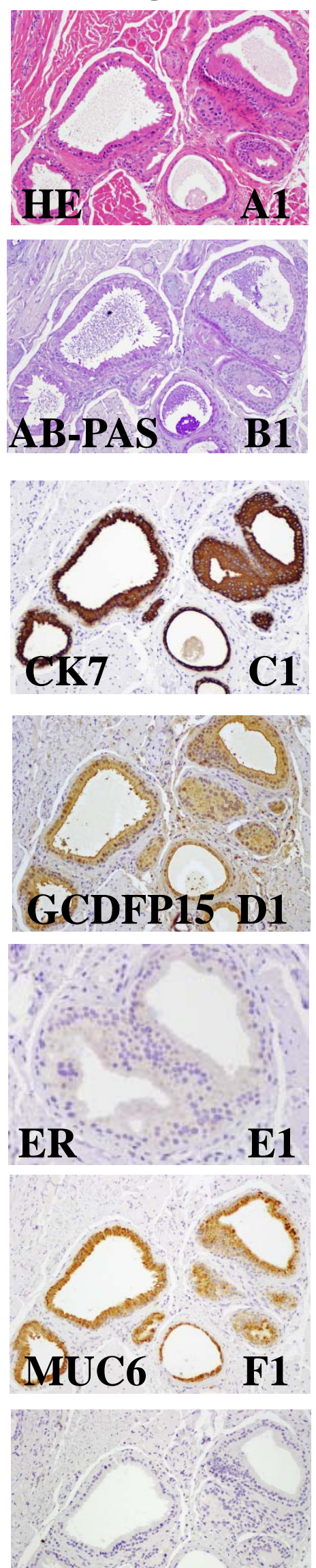

Apocrine gland
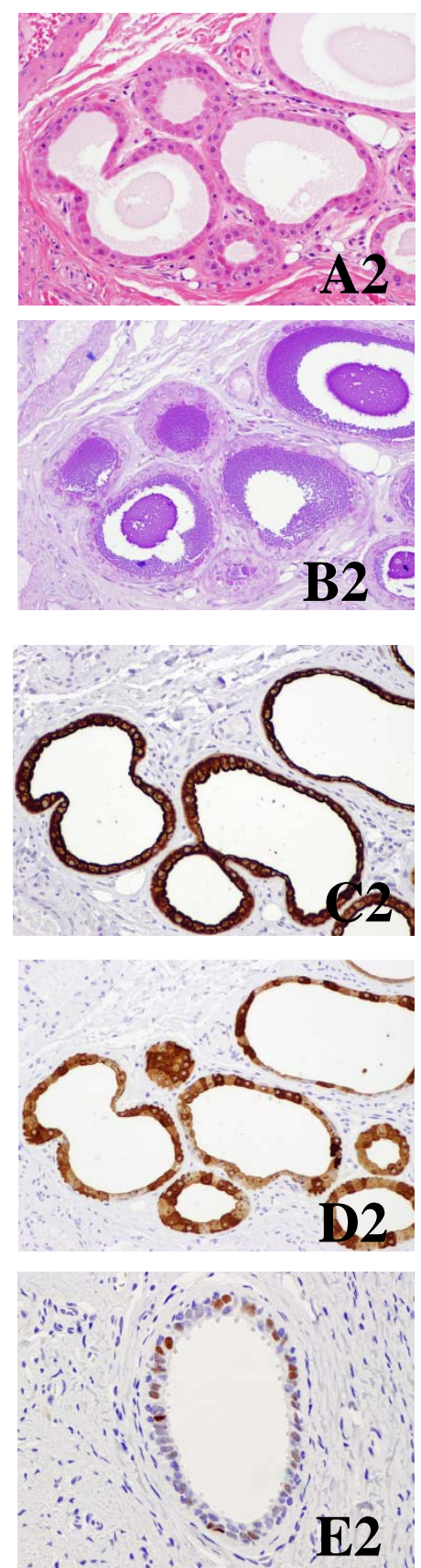

F2

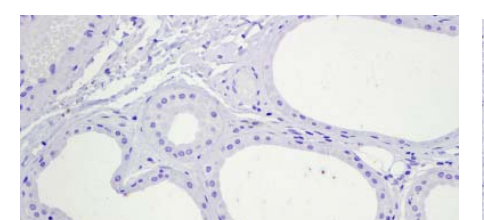

G2

\section{Eccrine gland}
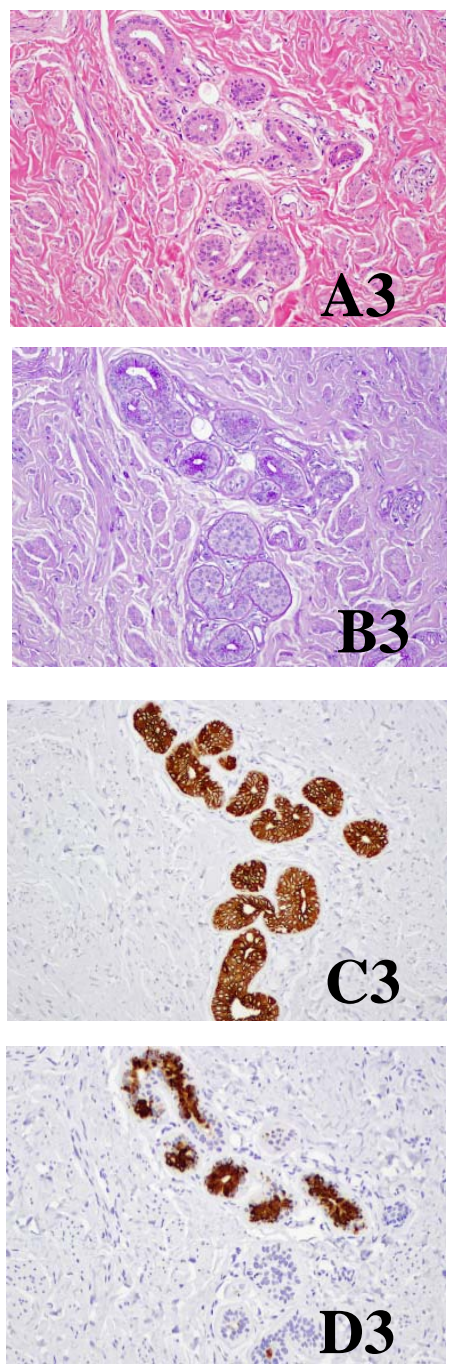

Fig 4 HIK1086 G1 